\title{
Characteristics of Asian 4 countries on cancer clinical trials registered in the International Clinical Trials Registry Platform between 2005 and 2018
}

\author{
Mariko Doi ${ }^{1}$, Keiko Yukawa ${ }^{1}$, Hajime Sato ${ }^{1,2} \wedge$ \\ ${ }^{1}$ Department of Health Policy and Technology Assessment, National Institute of Public Health, Minami 2-3-6, Wako, Saitama 351-0197, Japan; \\ ${ }^{2}$ Faculty of Global Nursing, Iryo Sosei University, Koaota 1-3-4, Kashiwa, Chiba 277-0803, Japan \\ Contributions: (I) Conception and design: M Doi, H Sato; (II) Administrative support: H Sato; (III) Provision of study materials or patients: M Doi; \\ (IV) Collection and assembly of data: M Doi; (V) Data analysis and interpretation: M Doi, H Sato; (VI) Manuscript writing: All authors; (VII) Final \\ approval of manuscript: All authors. \\ Correspondence to: Hajime Sato, MD, DPH, PhD. Department of Health Policy and Technology Assessment, National Institute of Public Health, \\ Minami 2-3-6, Wako, Saitama 351-0197, Japan. Email: hsato-tky@umin.ac.jp.
}

Background: The characteristics and comparison of countries regarding clinical trials for cancer were
unknown. The World Health Organization-International Clinical Trials Registry Platform (WHO-ICTRP)
are providing data from various countries and releases them generally in a downloadable format. We aimed
to examine and descriptively identify the number of cancer clinical trials registered in the world and the
fundamental characteristics in Asian 4 countries of China, India, Japan and South Korea, focusing on study
characteristics, e.g., phase or targeted size, using the WHO-ICTRP.

Methods: We extracted information on cancer clinical studies in the ICTRP database on September 20, 2019. Then, we performed a cross-sectional study on the annual number of registered studies and country, registered registry, phase, target sizes and sponsors.

Results: We identified 80,677 cancer clinical studies. The annual number of registered cancer clinical studies significantly increased between 2005 and 2018 (3,172 to 8,156, $\beta=382.2,95 \%$ CI: 329.6, 434.8). Among the Asian 4 countries, the numbers of trials were significantly increasing in $2005-2018(\mathrm{P}<0.001)$. The characteristics on interventional studies for cancer differed in those 4 countries, e.g., the registered registries, phase and sponsors, compared with the US.

Conclusions: This study descriptively clarified an increase and the characteristics of cancer clinical trials in Asian 4 countries. It suggests to take that characteristics into account and select a database or data source discreetly for analysis of clinical trials, according to the purpose of the research and the required data.

Keywords: Clinical trials; cancer; oncology; Clinical Trial Registry; International Clinical Trials Registry Platform (ICTRP)

Submitted Feb 09, 2021. Accepted for publication May 17, 2021.

doi: $10.21037 / \mathrm{cco}-21-17$

View this article at: http://dx.doi.org/10.21037/cco-21-17

\section{Introduction}

Cancer have been still the leading causes of death globally, thus highlighting the need for public health approaches to combat this health issue (1). For improving outcomes of patients with cancer, innovative treatments and treatment equipment for cancer are developing and evaluating. Clinical trials are fundamental platforms for the development of clinical treatments and guidelines, and practices for cancer management.

^ ORCID: Hajime Sato, 0000-0003-0796-0189; Mariko Doi, 0000-0002-8417-6147. 
The World Health Organization (WHO) launched the International Clinical Trials Registry Platform (ICTRP) in 2006 to consolidate data on trials registered in national and regional registries worldwide, thus providing a single point of access for all clinical trials registered globally. Collected data items differ depending on the primary registry but include at least the data items recommended in the WHO Trial Registration Data Set (TRDS), is publicly available in the registered record for all trials (2). The ICTRP integrates data from various countries and releases them generally in a downloadable format.

There have been many analyses on clinical trials, however most of them were studies using data of the regulatory agency or a clinical trial registry, e.g., ClinicalTrials.gov, UMIN-CTR (3-11). It was limited to a few studies by using ICTRP that focused on specific countries, organs or comprehensive study (12-15). Furthermore, the feature of countries regarding clinical trials for cancer were unknown. In this study, we aimed to examine and descriptively identify the number of cancer clinical trials registered in the world and the fundamental characteristics in Asian 4 countries of China, India, Japan and South Korea, focusing on study characteristics, e.g., phase or targeted size.

\section{Methods}

\section{ICTRP registry}

The ICTRP was established by the WHO in 2006. The information/data from each trial on the ICTRP website consists of the items detailed in the WHO Trial Registration Data Set (2). At the time that the present study was conducted, 17 national and regional clinical trial registries in Africa, Asia, Europe, Latin America and the Caribbean, Northern America, and Oceania had provided data on registered clinical trials to the ICTRP.

\section{Extraction of data from the ICTRP}

This cross-sectional study was performed descriptively. First, we performed an advanced search of the ICTRP database on September 20, 2019. After inputting the disease name in the condition column, data for the corresponding clinical trials were retrieved. In this study, we entered "Neoplasia or Neoplasm or Tumor or Cancer or Malignancy or Malignant" in the condition field. "ALL" was selected as the recruitment status field for the advanced search, and we subsequently analyzed the data from all clinical trials that were retrieved from this search. Of the clinical trials registered with the ICTRP (2), we acquired 80,677 data sets for analysis. The registered records for these studies were downloaded in XML format. For studies that were registered in more than one registry ("duplicate" registration), we included only the record with the earliest registration date (all ICTRP data that are publicly available are automatically "deduplicated", meaning that duplicate records of trials that were registered in more than one registry were removed). Among the identified studies, we included those with dates of registration between January 1, 2005 and December 31, 2018 and further subdivided them into observational and interventional studies based on the entries in the "Study Type" field. The clinical study data obtained from the above search criteria included all studies from three categories consists of intervention trials, observational studies and others based on WHO-TRDS.

\section{Statistical analysis}

Using descriptive analysis, we assessed the number and proportion of the following items to characterize and evaluate trends from 2005 (the earliest year of registration of a trial in one of the registries included in the ICTRP database) to 2018: (I) cancer interventional studies, (II) noninterventional studies for cancer, and (III) interventional studies for cancer recruited in the Asian 4 countries of China, India, Japan, South Korea. Then, for further analysis of the interventional studies for cancer conducted in Asian countries mentioned above, and the United States (US) from 2016 to 2018, we investigated the following characteristics: the registered clinical trial registry, study phase, estimated target size, and the type of sponsorship (industrial, e.g., pharmaceutical/bioresearch company or non-industrial, e.g., hospitals, university or researcher). Cochran-Armitage trend tests and Chi-squared were used to compare categorical and continuous variables on oncology trials, respectively. The aforementioned analyses were conducted using the $\mathrm{R}$ statistical package, version 3.5.2 (16).

\section{Results}

In total, 80,677 cancer clinical studies were identified using the ICTRP. After the inclusion based on the registration date, 70,457 clinical studies on cancer were analyzed for trends in the ICTRP registration. 


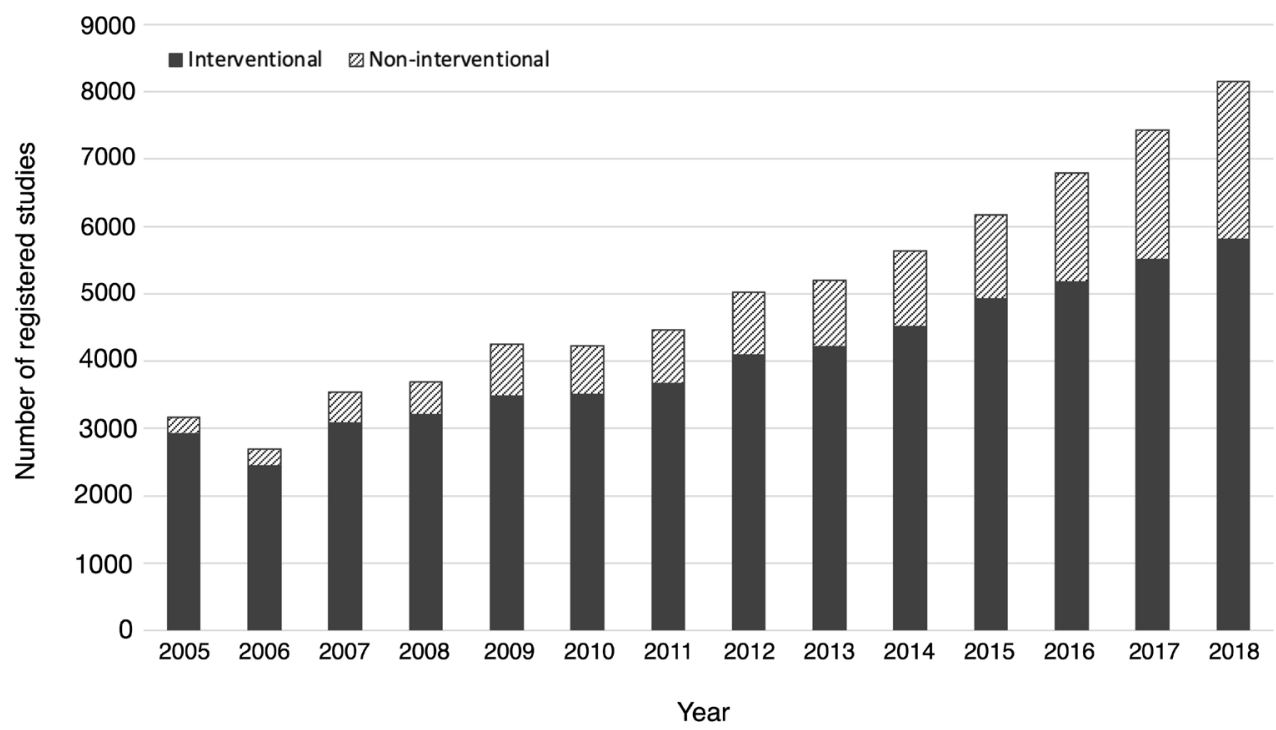

Figure 1 The annual number of registered studies in the International Clinical Trials Registry Platform from 2005 to 2018.

\section{The annual number of registered cancer clinical trials}

The registration trends were analyzed among 56,562 characterized interventional studies and 13,895 noninterventional studies. The annual number of the registered cancer trials in 2005-2018 are summarized in Figure 1. The number of registered clinical studies increased substantially, and there was more than two-fold increase between 2005 and 2018 (3,172 to 8,156 studies). The number of registered interventional and non-interventional studies increased an approximately two-fold (2,922 to 5,815 trials) and nine-fold (250 to 2,341 studies), respectively. The associations between the number of registered clinical trials and year of registration were assessed by a simple linear regression analysis, which revealed a significant association between the number of registered clinical trials and year of registration [interventional studies: $\beta=241.5,95 \%$ CI (14): 211.7, 271.2, adjusted $r^{2}=0.960$; all cancer clinical studies: $\beta$ $=382.2,95 \%$ CI: 329.6, 434.8, adjusted $\left.\mathrm{r}^{2}=0.951\right]$.

\section{The number of conducted cancer clinical trials by 4 country in Asia}

After the exclusion of 13,895 non-interventional studies, 56,562 interventional studies remained. Between 2005 and 2018, the highest number of cancer clinical trials registered in the ICTRP were enrollment in the US (19,392 trials), followed by Japan (8,429 trials). The number of cancer clinical trials conducted in Asian countries was as follows:
China (5,233 trials), South Korea (2,919 trials), and India (1,493 trials). Trends for the cancer interventional studies conducted in conducted countries which consist of India, South Korea, Japan and China, and were analyzed from 2005 to 2018 (Figure 2). Among the above mentioned Asian 4 countries, the numbers of trials were significantly increasing (India vs. Except India, $\mathrm{P}<0.001$; South Korea vs. Except South Korea, $\mathrm{P}<0.001$; Japan vs. Except Japan; China vs. Except China, $\mathrm{P}<0.001)$.

\section{Comparisons of characteristics on cancer clinical trials in Asian 4 countries and the US}

Details, e.g., source register or study phase of the study, of the cancer clinical trials within 4 countries in Asia and the US in the last three years were described to clarify the characteristics of Asian countries (Table 1). Clinical trials conducted in China, South Korea and the US had the highest number of registrations on ClinicalTrials.gov (China, 61.6\%, South Korea, 62.9\%, the US, 88.5\%). Clinical trials conducted in China and South Korea were also less registered in their domestic registries. The registry with the second highest number of registrations for clinical trials conducted in China was a China-based registry (34.1\%), while in South Korea the second most commonly used registry was EU-CTR as of European registry (15.1\%). Conversely, a high proportion of clinical trials conducted in India, and Japan were registered in their own registries, e.g., 


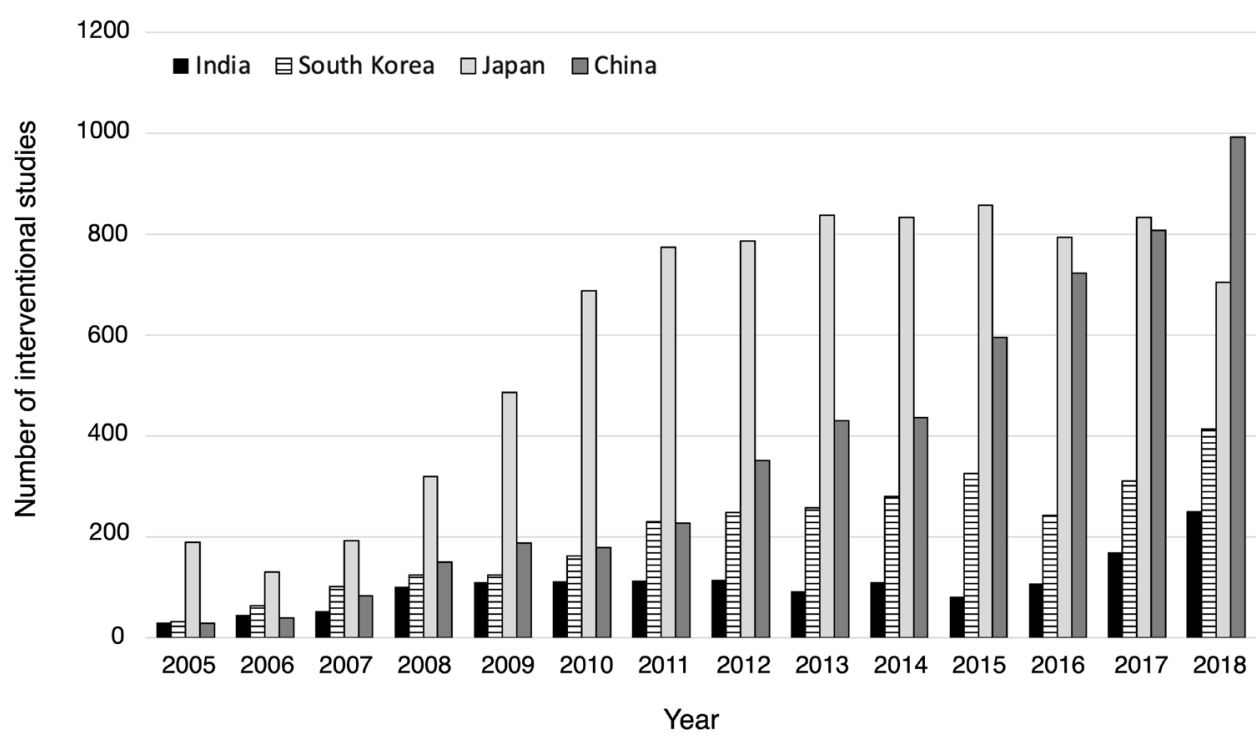

Figure 2 Distribution of interventional studies for cancer conducted in Asian 4 countries which were registered during 2005-2018 from ICTRP.

Clinical Trials Registry-India or Japan primary registries network each (India, 76.8\%; Japan, 78.7\%). In India and Japan, the second most commonly used clinical trial registry was ClinicalTrials.gov (India, 15.0\%, Japan, 13.3\%).

In 4 countries in Asia, the majority of the trials were in phase 2 trials and phase 3 trials (phase 2, 1,239 trials; phase 3, 1,235 trials). In each 4 countries of Asia, a greater number of phase 3 trials were conducted (China, 15.6\%, India, $28.3 \%$, Japan, $15.7 \%$, South Korea, $33.8 \%$, the US, $12.1 \%)(\mathrm{P}<0.001$ vs. the US, respectively), whereas lower proportion of phase 1 and phase 2 trials took place compared to in the US (phase 1, China, 10.3\%, India, 2.1\%, Japan, $8.7 \%$, South Korea, $8.2 \%$, the US, $21.8 \%$; phase 2, China, 20.6\%, India, 10.8\%, Japan, 17.1\%, Korea, 27.3\%, the US, $30.3 \%$ ) (phase $1, \mathrm{P}<0.001$ vs. the US, respectively; phase 2, $\mathrm{P}<0.001$, China, India and Japan vs. the US, respectively, $\mathrm{P}=0.069$, South Korea $v s$. the US). In India and Japan, high proportion of the category of "Others" were also selected (India, 39.2\%, Japan, 52.3\%) ( $\mathrm{P}<0.001$ vs. the US).

Around half of the trials conducted in the US and Japan had smaller for targeted sample size with $1-50$ cases (sum of 1-10 cases and 11-50 cases) (Japan, 49.4\%, the US, $45.3 \%)$. In contrast, in South Korea, and India, trials with over 100 cases (sum of $101-1,000$ cases and $>1,000$ cases) accounted for more than $40 \%$ of all trials (Korea, $41.2 \%$, India, $41.1 \%$ ), which were significantly higher proportion
$(\mathrm{P}<0.001$ vs. the US, respectively).

Concerning the sponsorship, South Korea had a significantly higher proportion of industrial sponsorship than the US (South Korea, 56.7\%, the US, 38.1\%) $(\mathrm{P}<0.001$ $v s$. the US). Conversely, China, India and Japan had a lower proportion of industrial sponsorship (China, 19.4\%, India, $30.0 \%$, Japan, $22.5 \%$ ) (China, $\mathrm{P}<0.001$, India, $\mathrm{P}=0.405$, Japan, $\mathrm{P}<0.001)$.

\section{Discussion}

The present study investigated the cancer clinical trials of the world registered in the clinical trial registries and the characteristics and trends in these trials in Asian 4 countries of China, India, Japan and South Korea. Previous studies on cancer clinical trials have only reported on studies included in specific countries or organs $(5,7,9,14)$. However, none have involved comprehensive evaluation or comparison between countries. This study demonstrated an increase in the annual number of cancer clinical trials by utilizing the WHO-ICTRP and revealed the characteristics of cancer clinical trials conducted in those 4 countries.

Our findings are consistent with those reported in previous studies in which there was an annual increase in the number of trials registered in ICTRP (15). Previous studies were not specific to cancer and thus our findings are novel in that they report on cancer trials. In this study, 
Table 1 Characteristics of intervention studies for cancer conducted in Asian 4 countries on ICTRP in 2016-2018

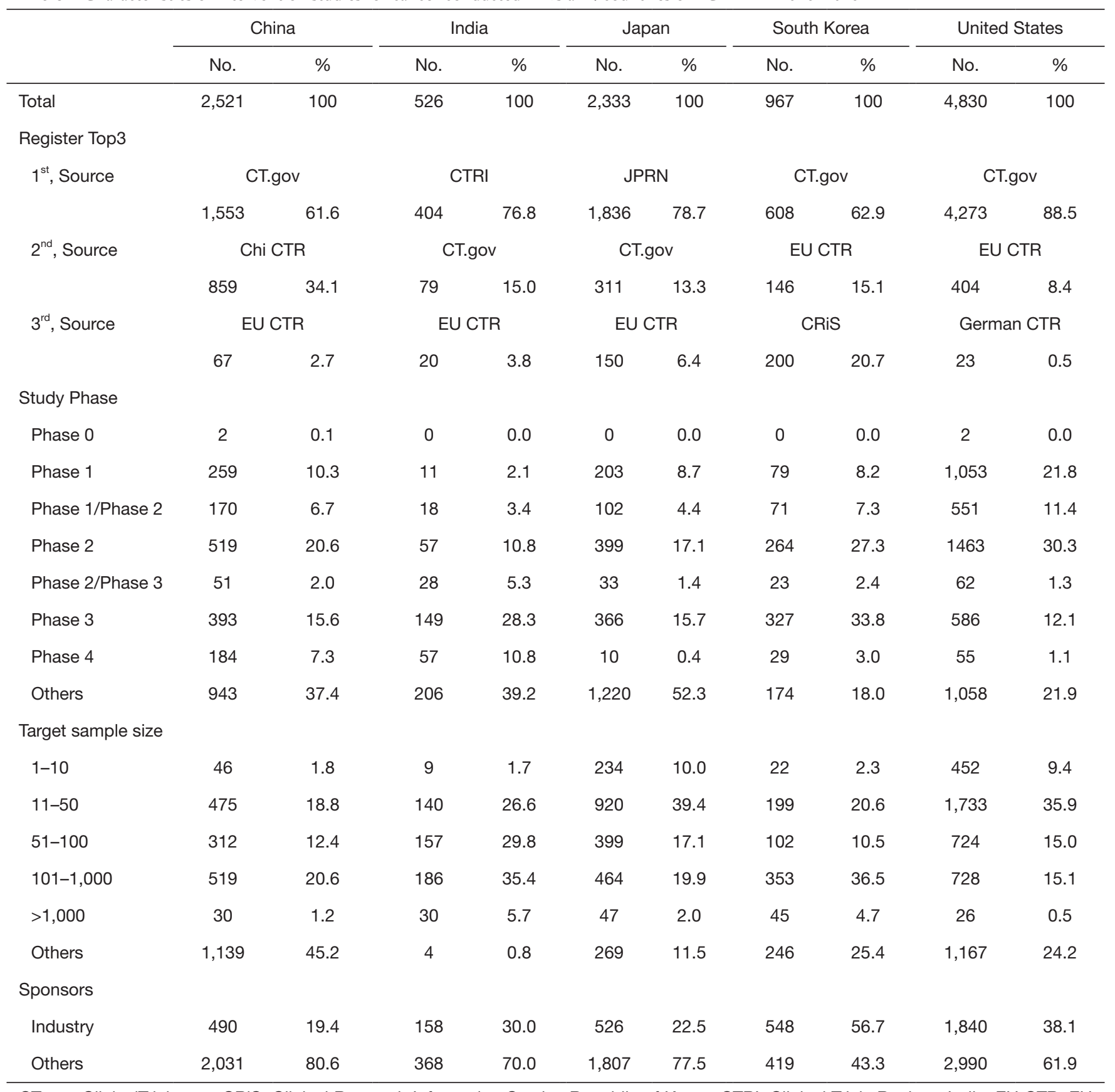

CT.gov, ClinicalTrials.gov; CRiS, Clinical Research Information Service Republic of Korea; CTRI, Clinical Trials Registry-India; EU CTR, EU Clinical Trials Register; German CTR, German Clinical Trials Register; JPRN, Japan Primary Registries Network.

the numbers of cancer clinical trials were increasing between 2005 and 2018 both in the world and in the Asian 4 countries. Other studies found that the number of cancer clinical trials initiated and approved by the regulatory affairs increased in China and South Korea $(5,9,17)$. In the present study, the number of cancer clinical trials conducted in China and South Korea was also increasing during 2005 and 2018, suggesting that the growth number of clinical trials for cancer might be led to have increased the number of approvals. The number of cancer clinical trials conducted 
in India and Japan were also increasing in this study. Some studies have been indicated that Asian countries have grown rapidly to be conducted the clinical trials, suggested that they have potential to grow into major players in previous studies $(6,18)$. The increase in the number of registered cancer clinical trials of Asian 4 countries in this study is also consistent with those results and can be seen as reflecting the annual increase of the conducted cancer clinical trials in Asia. The effects for the number of trials in Asian countries are thought to be the followings. At first, the increased cancer patients in Asia might be considered. The agestandardized incidence rate on cancer is growing (19), and it may be thought that the number of cancer patients is increasing. The large number of untreated patients contributes significantly to the implementation of clinical trials. The increasing number of cancer patients also may contribute the development of patients' medical knowledge and their willingness to participate in clinical trials. Moreover, the number of patients, and even the number of clinical trials, would be attributed to the size of the country's population. Secondly, operating costs on clinical trials in Asia were also considered to affect. In industrial clinical trials, clinical trial costs in Asia are also considered to be lower than in the United States and western Europe $(20,21)$. The advantages of lower operational costs might be thought as contributing factors to increase the number clinical trials. Thirdly, the improvement of environment to conduct clinical trials could contribute, e.g., centers or sites generate high-quality data $(5,22)$. In South Korea and China, it was thought that the clinical trials are being promoted that patients are aggregated and pooled, and that the system is efficiently set up to influence clinical research $(9,17,22)$. Finally, the dissemination of EBM concepts or implementation of the International Conference on Harmonization-Good Clinical Practice (ICH-GCP), the improvement of regulatory environment $(17,20,23,24)$. In addition, it might be also possible that the number of clinical trial registrations has increased due to the widespread understanding of clinical trial registration.

The characteristics on cancer clinical trials conducted in Asian 4 countries differed among countries in 2016-2018, e.g., the registered registries for cancer clinical trials. In China and South Korea, most of the cancer clinical trials conducted were registered with ClinicalTrials.gov. In contrast, most of cancer clinical trials conducted in Japan and India were not. The high proportion of its domestic registry in India was reported in the previous studies (8). Most of the studies being conducted in India might be registered in the Indian domestic registry. The registration trends in those registries varied among countries, therefore it should be considered that the characteristics on data in ClinicalTrials.gov and ICTRP for data analysis on cancer clinical trials. Previous study also reported the necessity of searching ICTRP in addition to ClinicalTrials.gov for systematic reviewers (25).

In Asian 4 countries, there were many studies in phase, 3 , and in the US, there were many studies in early phases, e.g., phase 1. Prior study reported that the trend in Asian countries is with phase III clinical trials, not for early stage/ phase I clinical trials $(6,7,11)$. In this study, we also found that trials with small sample sizes, e.g., 1-50 and early phases, e.g., phase 1 and 2 were frequently conducted in the US.

The majority of the trials enrollment in Korea and the US were sponsored by the industries; however, many clinical trials in Japan and China are sponsored by others (non-industries). Due to the fact that industry-sponsored clinical trials are more common in the US and South Korea, this may be explained by the high proportion of studies registered in ClinicalTrials.gov and EU-CTR that are registries of clinical trials for investigational new drug. In this study, it was clarified the characteristics of cancer clinical trials in Asian 4 countries. Asian countries had the different characteristics. In China, the number of clinical trials has strikingly increased, and there was a possibility that the trend will continue to increase in the future (20). There were a lot of investigator-sponsored clinical trials and trial phase was relatively balanced (22). In India, many large-scale trials with late phase were conducted (6). In contrast, there are many small-scale trials with unclear of the phase in Japan, and there might be many trials on new treatment in clinical practice by clinicians. The number of clinical trials was large, but its increase rate has almost disappeared. In Korea, there are many trials conducted with large-scale, industrial sponsored, at phase 2 and 3 (9).

Our findings lead to a recommendation for the research on clinical trials in Asian 4 countries. The findings suggest considering the characteristics on data in ICTRP and national registry, e.g., ClinicalTrials.gov, for the research. There were founded several variations of cancer clinical trials of Asian 4 countries in this study, e.g., registered registry, phase, sponsor. It is necessary to select a database or data source according to the purpose of the research, and ICTRP might be one of the candidates in those cases.

The strength of the present study was that the data registered in the ICTRP was utilized, thus allowing us to evaluate the number of overall cancer clinical trials 
conducted globally. Despite these strengths, this study was subject to several limitations. First, the type of intervention was not considered. While there are many types of clinical trials on cancer, such as drug treatment and surgery, diagnosis and prevention, and palliative care, the present study assessed aggregate data on cancer clinical trials. Depending on the type of intervention, factors such as the phase, sample size, and funding source might differ; however, this was not discussed in the current study. Therefore, further analysis might be required depending on the type of intervention type, target organ, and the study purpose. Second, the quality of data may vary among those registries, and not only from country to country $(15,26)$. If there were many missing data, completeness of data might have affected the analysis. In addition, the accuracy of the data also affects the results. Differences in quality control and its procedures $(8,26,27)$ and changes of the registry management systems might affect the quality of data on each registry. Therefore, differences in registered registries may affect inter-country comparisons, and it is necessary to pay attention carefully when handling and analyzing ICTRP data in the future. In a few items that not analyzed in this study, there were uncertain items on the data format. Thus, it would be desirable of continuous quality control in each registry, and standardization, cooperation of registered data inter-registry in the future (26). However, in this study, we could not find that there was obviously difference on items reported in this article between the registries with the exception of the unknown phase in Japanese studies.

\section{Conclusions}

In conclusion, our study descriptively clarified an increase and the characteristics of interventional studies for cancer in Asian 4 countries. It suggests taking that characteristics into account and select a database or data source discreetly for analysis of clinical trials, according to the purpose of the research and the required data. In the future, it is desirable to conduct a detailed study based on the differences between the target regions and the content of the interventions.

\section{Acknowledgments}

We acknowledge Dr. Masafumi Okada for his valuable assistance with ascertaining and analyzing the data from the ICTRP.

Funding: None.

\section{Footnote}

Conflicts of Interest: All authors have completed the ICMJE uniform disclosure form (available at http://dx.doi. org/10.21037/cco-21-17). The authors have no conflicts of interest to declare.

Ethical Statement: The authors are accountable for all aspects of the work in ensuring that questions related to the accuracy or integrity of any part of the work are appropriately investigated and resolved.

Open Access Statement: This is an Open Access article distributed in accordance with the Creative Commons Attribution-NonCommercial-NoDerivs 4.0 International License (CC BY-NC-ND 4.0), which permits the noncommercial replication and distribution of the article with the strict proviso that no changes or edits are made and the original work is properly cited (including links to both the formal publication through the relevant DOI and the license). See: https://creativecommons.org/licenses/by-nc-nd/4.0/.

\section{References}

1. WHO Cancer [Internet]. World Health Organization; c2020 [cited 2020 Aug 16]. Available online: https://www. who.int/cancer/en/

2. WHO International Clinical Trials Registry Platform (ICTRP) [Internet]. World Health Organization; c2020 [cited 2020 Aug 16]. Available online: https://www.who. int/ictrp/en/

3. Califf RM, Zarin DA, Kramer JM, et al. Characteristics of clinical trials registered in ClinicalTrials.gov, 2007-2010. JAMA 2012;307:1838-47.

4. Jeong S, Sohn M, Kim JH, et al. Current globalization of drug interventional clinical trials: characteristics and associated factors, 2011-2013. Trials 2017;18:288.

5. Li N, Huang HY, Wu DW, et al. Changes in clinical trials of cancer drugs in mainland China over the decade 200918: a systematic review. Lancet Oncol 2019;20:e619-26.

6. Louisa M, Takeuchi M, Setiabudy R, et al. Current status of phase I clinical trials in Asia: an academic perspectives. Acta Med Indones 2012;44:71-7.

7. Ochi N, Kawahara T, Nagasaki Y, et al. Publication of lung cancer clinical trials in the Japanese Clinical Trial Registry. Jpn J Clin Oncol 2018;48:995-1000.

8. Pillamarapu M, Mohan A, Saberwal G. An analysis of deficiencies in the data of interventional drug trials 
registered with Clinical Trials Registry - India. Trials 2019;20:535.

9. Shim BY, Park SH, Lee S, et al. Current Status and Challenges of Cancer Clinical Trials in Korea. Cancer Res Treat 2016;48:20-7.

10. Tang $W$, Fukuzawa $M$, Ishikawa $H$, et al. Review of the registration of clinical trials in UMIN-CTR from 2 June 2005 to 1 June 2010 - focus on Japan domestic, academic clinical trials. Trials 2013;14:333.

11. Yang JJ, Wu YL. Insight into early-phase trials for lung cancer in the United States. Chin J Cancer 2015;34:288-94.

12. Atal I, Trinquart L, Porcher R, et al. Differential Globalization of Industry- and Non-Industry-Sponsored Clinical Trials. PLoS One 2015;10:e0145122.

13. Feizabadi M, Fahimnia F, Mosavi Jarrahi A, et al. Iranian clinical trials: An analysis of registered trials in International Clinical Trial Registry Platform (ICTRP). J Evid Based Med 2017;10:91-6.

14. Lee B, Cuervo LG, Rodriguez-Feria P, et al. Analysis of registered cancer clinical trials in Latin America and the Caribbean, 2007-2013. Rev Panam Salud Publica 2016;39:115-21.

15. Viergever RF, Li K. Trends in global clinical trial registration: an analysis of numbers of registered clinical trials in different parts of the world from 2004 to 2013. BMJ Open 2015;5:e008932.

16. R Core Team R: A language and environment for statistical computing. R Foundation for Statistical Computing, Vienna, Austria; c2020 [cited 2020 Aug 16]. Available online: https://www.R-project.org/

17. Im SA, Lee DW, Kang R, et al. Challenges and insights of early oncology drug development in the Asia-Pacific region: introduction of phase I oncology clinical trial

Cite this article as: Doi M, Yukawa K, Sato H. Characteristics of Asian 4 countries on cancer clinical trials registered in the International Clinical Trials Registry Platform between 2005 and 2018. Chin Clin Oncol 2021;10(3):28. doi: 10.21037/cco-21-17 center and experience sharing for early clinical trials in Seoul National University Hospital, Korea. Chin Clin Oncol 2019;8:27.

18. Thiers FA, Sinskey AJ, Berndt ER. Trends in the globalization of clinical trials. Nat Rev Drug Discov 2008;7:13-4.

19. International Agency for Research on Cancer [Internet]. Lyon: World Health Organization [cited 2021 Apr 12]. Global Cancer Observatory. Available online: https://gco. iarc.fr/

20. Nicholas J. Outsourcing clinical trials. J Natl Cancer Inst 2012;104:1043-5.

21. Frost and Sullivan Inc. ASIA: Preferred Destination For Clinical Trials. 2020.

22. Wu YL, Zhang H, Yang Y. Cancer drug development in China: recent advances and future challenges. Drug Discov Today 2015;20:766-71.

23. Wang M. Clinical trials and drug approvals continue to accelerate in China. Lancet Oncol 2017;18:855.

24. Ono S, Kato O, Tsutani K, et al. Utilisation of Foreign Clinical Data in Japanese New Drug Approval Review. International Journal of Pharmaceutical Medicine 2004;18:159-65.

25. Banno M, Tsujimoto Y, Kataoka Y. Studies registered in non-ClinicalTrials.gov accounted for an increasing proportion of protocol registrations in medical research. J Clin Epidemiol 2019;116:106-13.

26. Viergever RF, Karam G, Reis A, et al. The quality of registration of clinical trials: still a problem. PLoS One 2014;9:e84727.

27. World Health Organization. International standards for clinical trial registries. Geneva: World Health Organization; 2012. 